\title{
DECOMPOSITION OF HARDY FUNCTIONS INTO SQUARE INTEGRABLE WAVELETS OF CONSTANT SHAPE*
}

\author{
A. GROSSMANN ${ }^{\dagger}$ AND J. MORLET ${ }^{\ddagger}$
}

\begin{abstract}
An arbitrary square integrable real-valued function (or, equivalently, the associated Hardy function) can be conveniently analyzed into a suitable family of square integrable wavelets of constant shape, (i.e. obtained by shifts and dilations from any one of them.) The resulting integral transform is isometric and self-reciprocal if the wavelets satisfy an "admissibility condition" given here. Explicit expressions are obtained in the case of a particular analyzing family that plays a role analogous to that of coherent states (Gabor wavelets) in the usual $L_{2}$-theory. They are written in terms of a modified $\Gamma$-function that is introduced and studied. From the point of view of group theory, this paper is concerned with square integrable coefficients of an irreducible representation of the nonunimodular $a x+b$-group.
\end{abstract}

\section{Introduction.}

1.1. It is well known that an arbitrary complex-valued square integrable function $\psi(t)$ admits a representation by Gaussians, shifted in direct and Fourier transformed space. If $g(t)=2^{-1 / 2} \pi^{-3 / 4} e^{-t^{2} / 2}$ and $t_{0}, \omega_{0}$ are arbitrary real, consider

$$
g^{\left(t_{0}, \omega_{0}\right)}(t)=e^{-i \omega_{0} t_{0} / 2} e^{i \omega_{0} t} g\left(t-t_{0}\right)
$$

and form the inner product

$$
\Psi\left(t_{0}, \omega_{0}\right)=\int \bar{g}^{\left(t_{0}, \omega_{0}\right)}(t) \psi(t) d t
$$

Then

$$
\iint\left|\Psi\left(t_{0}, \omega_{0}\right)\right|^{2} d t_{0} d \omega_{0}=\int|\psi(t)|^{2} d t
$$

The function $\psi(t)$ can be recovered from the function $\Psi\left(t_{0}, \omega_{0}\right)$ through

$$
\psi(t)=\iint g^{\left(t_{0}, \omega_{0}\right)}(t) \Psi\left(t_{0}, \omega_{0}\right) d t_{0} d \omega_{0}
$$

The above statements remain true if the Gaussian $g$ is replaced by an arbitrary square integrable function. The advantages of the Gaussian are (i) maximal concentration in direct and Fourier transformed space and (ii) the possibility of a simple intrinsic characterization of the space of functions $\Psi\left(t_{0}, \omega_{0}\right)$.

This representation of functions has been used in quantum mechanics, quantum optics and signal theory. (See e.g. [1], [4], [5], [6].)

1.2. Consider now the case where the object of interest is not a complex-valued function $\psi(t)$, but a square integrable real-valued function $s(t)$, say the wiggle of a seismograph. It has been known for a long time that it is very useful to consider $s(t)$ as the real part of a complex-valued square integrable function $h(t)$ which has the special property that its Fourier transform vanishes on a half-line (say $\tilde{h}(\omega)=0$ for $\omega<0$ ). The

* Received by the editors September 2l, 1982.

${ }^{\dagger}$ Centre de Physique Theorique, Section II, Centre National de la Recherche Scientifique, Marseille, France.

‡Elf Aquitaine Company, O.R.I.C. Lab., 370 bis Av. Napoléon Bonaparte, 92500 Rueil-Malmaison, France. 
space of such functions $h(t)$ is denoted by $\mathbf{H}^{2}$ and called the Hardy space on the line. It is a closed subspace of the space $L_{2}(\mathbb{R}, d t)$ of all square integrable functions. The functions $s$ and $h$ are in a natural one-to-one correspondence, and special properties of the function $h(t)$ (in particular its phase) make it a valuable tool.

1.3. This paper is concerned with the decomposition of functions $h \in \mathrm{H}^{2}$ into square integrable "elementary wavelets", and with the corresponding reconstruction problem. One can of course analyze the function $h(t)$ by applying to it the general results described in $\S 1.1$, applicable to any function in $L_{2}$. This is indeed what is done traditionally (see e.g. the famous paper [4]). It is however clear that, when we follow this procedure, we are not taking advantage of the special features of the function $h(t)$ which led us to introduce it in the first place; we are analyzing a function that belongs to the subspace $\mathrm{H}^{2} \subset L_{2}$ in terms of wavelets that do not belong to this subspace (the Fourier transform of a Gaussian does not vanish on a half-line). It will not help (at least in principle) to replace the Gaussian by an elementary wavelet that belongs to $\mathbf{H}^{2}$, since we have to consider all of its shifts in Fourier transformed space, and these are sure to bring it out of $\mathbf{H}^{2}$.

1.4. In several papers devoted to the study of seismic traces [7], [8], one of us has suggested analyzing them in terms of wavelets of fixed shape, and has produced strong numerical evidence for the soundness of such analysis. The aim of the present paper is to give mathematical underpinnings for this procedure, which also avoids the objections that were raised in $\S 1.3$. The main idea is to analyze functions in terms of wavelets obtained by shifts (only in direct space, not in Fourier transformed space) and dilations from a suitable basic wavelet.

1.5. The group $G_{2}$ of shifts and dilations (which is the only two-parameter Lie group and thus the "smallest" noncommutative Lie group), acts on $\mathbf{H}^{2}$ through a natural irreducible unitary representation $U(\gamma)\left(\gamma \in G_{2}\right)$. If we fix a function $g \in \mathbf{H}^{2}$ ("the analyzing wavelet"), we obtain a correspondence between an arbitrary $h \in \mathbf{H}^{2}$, and the matrix element $m_{h}^{(g)}(\gamma)=(U(\gamma) g, h)$ considered as a function on the group $G_{2}$. The main question, both from a conceptual and practical point of view, is whether the correspondence $h \rightarrow m_{h}^{(g)}$ has a well-behaved inverse, allowing a "stable" reconstruction of $h$ from $m_{h}^{(g)}$. Stated somewhat differently, the question is whether, for a suitable invariant measure $d \gamma$ on $G_{2}$, one has

$$
\int\left|m_{h}^{(g)}(\gamma)\right|^{2} d \gamma=\int|h(t)|^{2} d t
$$

in analogy to (1.3).

1.6. It turns out that the answer depends on the choice of the analyzing wavelet $g$. For (1.5) to hold, the wavelet $g$, in addition to being in $\mathbf{H}^{2}$, has to satisfy an "admissibility" condition.

The main general result, proved in $\S 3$, can be stated without reference to group theory:

Let $h(t)$ (the function to be analyzed) satisfy

$$
\int|h(t)|^{2} d t<\infty
$$


and

(ii)

$$
\tilde{h}(\omega)=0 \text { for } \omega \leq 0
$$

(Conditions (i) and (ii) say that $h \in \mathbf{H}^{2}$.)

Let $g(t)$ (the analyzing wavelet) satisfy (i), (ii), and also the "admissibility condition"

(iii) $\int d u e^{u} \int_{0}^{\infty}\left|\tilde{g}(\omega) \tilde{g}\left(e^{u} \omega\right)\right|^{2} d \omega<\infty$. Associate to $h$ the function $(e h)(u, v)$ of two variables, defined by

$$
(\bigodot h)(u, v)=\frac{1}{\sqrt{c_{g}}} e^{u / 2} \int \bar{g}\left(e^{u} t-v\right) h(t) d t
$$

where

$$
c_{g}=\frac{2 \pi}{\|g\|^{2}} \int d u e^{u} \int\left|\tilde{g}(\omega) \tilde{g}\left(e^{u} \omega\right)\right|^{2} d \omega=2 \pi \int_{0}^{\infty} \frac{|\tilde{g}(\omega)|^{2}}{\omega} d \omega
$$

and

$$
\|g\|^{2}=\int|g(t)|^{2} d t
$$

Then

(a)

$$
\iint|(e h)(u, v)|^{2} d u d v=\int|h(t)|^{2} d t
$$

and

(b) $h(t)$ can be recovered from $(e h)(u, v)$ through

$$
h(t)=\frac{1}{\sqrt{c_{g}}} \iint e^{u / 2} g\left(e^{u} t-v\right)(\bigodot h)(u, v) d u d v
$$

If $g$ is not admissible, then $c_{g}=\infty$, and the transformation (1.6) is not defined.

The need for an admissibility condition may seem surprizing. It stems from the fact that $G_{2}$, in contrast to all other "everyday" groups, is nonunimodular (i.e. has no right-and-left-invariant measure) (compare [2], [3]).

Section 4 is devoted to a more detailed study of the transformation $\mathcal{C}$ in the special case where $g$ is a "particularly good" wavelet which plays a role analogous to that of the Gaussian in the conventional theory. We find it convenient to introduce a special function $\Gamma_{\alpha}(z)$ which may be of independent interest. In $\$ 4$ we gather the results necessary for an intrinsic characterization of the range of $e$, which will be given in a forthcoming paper.

This paper can also be viewed as the description of a natural quantum-mechanical representation for particles that "only know how to move in one direction".

This interpretation and further developments will also be found in forthcoming papers. 


\section{Notation and preliminaries.}

2.1. The inner product of square integrable functions is written as

$$
(f, g)=\int \bar{f}(t) g(t) d t
$$

where $\bar{f}$ is the complex conjugate of $f$.

The Fourier transform of $f(t)$ is

$$
\tilde{f}(\omega)=(2 \pi)^{-1 / 2} \int e^{-i \omega t} f(t) d t
$$

inverted by

$$
f(t)=(2 \pi)^{-1 / 2} \int e^{i \omega t} \tilde{f}(\omega) d \omega .
$$

We also write

$$
\tilde{f}=\mathscr{F} f, \quad f=\mathscr{F}^{-1} \tilde{f} .
$$

The shift operator $T^{v}$ is defined by

$$
\left(T^{v} f\right)(t)=f(t-v) \quad(v \in \mathbb{R}) .
$$

The corresponding multiplication operator is $E^{v}$;

$$
\left(E^{v} \tilde{f}\right)(\omega)=e^{i \omega \omega} \tilde{f}(\omega) .
$$

The dilation operator $Z^{u}$ is defined by

$$
\left(Z^{u} f\right)(t)=e^{-u / 2} f\left(e^{-u} t\right) .
$$

The relations

$$
\begin{array}{rlrl}
T^{v} Z^{u} & =Z^{u} T^{v / \exp u}, & E^{v} Z^{u}=Z^{u} E^{v \exp u}, \\
Z^{u} T^{v}=T^{v \exp u} Z^{u}, & Z^{u} E^{v}=E^{v / \exp u} Z^{u}
\end{array}
$$

will be basic for all that follows. They correspond to

$$
\begin{aligned}
& \left(T^{v} Z^{u} f\right)(t)=e^{-u / 2} f\left(e^{-u t}-e^{-u} v\right), \\
& \left(Z^{u} T^{v} f\right)(t)=e^{-u / 2} f\left(e^{-u} t-v\right) .
\end{aligned}
$$

The commutation properties with $\mathscr{F}$ are

$$
\begin{aligned}
& \mathscr{F} T^{v}=E^{-v \mathscr{F}}, \\
& \mathscr{F} Z^{u}=Z^{-u \mathscr{F}} .
\end{aligned}
$$

We have $T^{v_{1}} T^{v_{2}}=T^{v_{1}+v_{2}}$, and $Z^{u_{1}} Z^{u_{2}}=Z^{u_{1}+u_{2}}$. The operators $\mathscr{F}, T^{v}, E^{v}, Z^{u}$ are unitary in $L_{2}(\mathbb{R})$.

2.2. We say that a function $h \in L_{2}(\mathbb{R}, d t)$ belongs to the Hardy space $\mathbf{H}^{2} \subset L$ $\tilde{h}(\omega)=0$ for $\omega<0$.

$\mathbf{H}^{2}$ is a closed subspace of $L_{2}$.

A real-valued function cannot belong to $\mathbf{H}^{2}$.

If $h \in \mathbf{H}^{2}$ then $\bar{h}$ and $\check{h}$ (where $\check{h}(t)=h(-t)$ ) are orthogonal to all of $\mathbf{H}^{2}$. Howe $h^{*} \in \mathbf{H}^{2}$, where $h^{*}(t)=\bar{h}(-t)$. 
The Hilbert transform in $L_{2}(\mathbb{R}, d t)$ can be defined as $H=-\mathscr{F}^{-1} \varepsilon^{\mathscr{F}}$, where $\varepsilon$ is the operator of multiplication by sgn $\omega$ (the sign of $\omega$ ). We have $H^{2}=-1 ; H$ is unitary, anti-Hermitian and real (commutes with complex conjugation). If $h \in \mathbf{H}^{2}$, then $h=i H h$, giving $\operatorname{Im} h=H \operatorname{Re} h$ and $\operatorname{Re} h=-H \operatorname{Im} h$.

If $s(t)$ is any real-valued, square integrable function then

$$
h_{s}=s+i H s
$$

belongs to $\mathbf{H}^{2}$. We have

$$
\left(h_{s}, g\right)=2(s, g)
$$

for every $g \in \mathbf{H}^{2}$. Also

$$
\left\|h_{s}\right\|^{2}=2\|s\|^{2}
$$

where $\|f\|^{2}=(f, f)$

2.3. Shifts and dilations in $\mathbf{H}^{2}$. If $h \in \mathbf{H}^{2}$, then $T^{v} h \in \mathbf{H}^{2}$ and $Z^{u} h \in \mathbf{H}^{2}$ for all $u, v$.

For our purposes it is crucial to remark that the family $T^{v}, Z^{u}$ acts irreducibly in $\mathbf{H}^{2}$. That is: If $V$ is a closed subspace of $\mathbf{H}^{2}$, containing at least one nonzero vector; if $V$ is stable under all $Z^{u}, T^{v}$ (which means $T^{u} Z^{v} h \in V$ whenever $h \in V$ ), then $V$ is all of $\mathrm{H}^{2}$. For a proof see e.g. [9].

Another important, if obvious, remark is that shifts and dilations are "real", in the sense that

$$
\operatorname{Re}\left(T^{v} h\right)=T^{v}(\operatorname{Re} h)
$$

and

$$
\operatorname{Re}\left(Z^{u} h\right)=Z^{u}(\operatorname{Re} h)
$$

\section{The $\mathcal{C}$-transform: arbitrary admissible wavelet.}

3.1. Admissible analyzing wavelet. We shall say that a function $g$, not identically zero, is an admissible analyzing wavelet, if

and

(i) $\mathrm{g}$ belongs to $\mathrm{H}^{2}$

(ii) $g$ satisfies the condition

$$
\iint\left|\left(Z^{-u} T^{v} g, g\right)\right|^{2} d u d v<\infty
$$

By (2.2) and (2.3), the condition (3.1) can also be written as

$$
\begin{aligned}
\iint\left|\left(Z^{u} E^{-v} \tilde{g}, \tilde{g}\right)\right|^{2} d u d v & =2 \pi \int d u e^{u} \int\left|\tilde{g}(w) \tilde{g}\left(e^{u} \omega\right)\right|^{2} d \omega<\infty \\
& =2 \pi\|g\|^{2} \int_{0}^{\infty} \frac{|\tilde{g}(\omega)|^{2}}{\omega} d \omega .
\end{aligned}
$$

Examples. 1) Let $0<a<b<\infty$. Define $g(t)$ through its Fourier transform: $\tilde{g}(\omega)=1$ if $a<\omega<b$, and 0 otherwise. Then $g(t)$ is an admissible analyzing wavelet, as can be shown by a simple calculation.

2) Let $\alpha>0$. Define $g_{\alpha}(t)$ through its Fourier transform $\tilde{g}_{\alpha}(\omega)=\exp \left(-(\alpha / 2) \ln ^{2} \omega\right)$ $=\omega^{-\alpha \ln \omega / 2}$ for $\omega>0$, and $g_{\alpha}(\omega)=0$ for $\omega<0$. Then $g_{\alpha}(t)$ is an admissible analyzing wavelet which will be studied in $\$ 4$. 
Remarks. 1) There exist functions in $\mathrm{H}^{2}$ that are not admissible analyzing wavelets; this is the case e.g. if the Fourier transform of $g$ is defined by

$$
\tilde{g}(\omega)=\left\{\begin{array}{ll}
\omega^{-1 / 2+\varepsilon} & (0<\omega \leq 1) \\
\omega^{-1 / 2-\varepsilon} & (1<\omega<\infty)
\end{array} \quad(\varepsilon>0)\right.
$$

and $\tilde{g}(\omega)=0$ for $\omega \leq 0$.

2) A Gaussian cannot be an admissible analyzing wavelet since it does not belong to $\mathbf{H}^{2}$. However, if $\omega_{0}$ is positive and sufficiently large, the function $e^{i \omega_{0} t} \exp \left(-t^{2} / 2\right)$ is very close to an admissible analyzing wavelet.

3) From (3.2) we see: If $\tilde{g}(\omega)$ is the Fourier transform of an admissible wavelet then, for any real-valued $\varphi(\omega)$, the function $e^{i \varphi(\omega)} \tilde{g}(\omega)$ is also the Fourier transform of an admissible wavelet. number

3.2. The number $c_{g}$. If $g$ is an admissible analyzing wavelet, we denote by $c_{g}$ the

$$
c_{g}=\frac{1}{\|g\|^{2}} \iint\left|\left(Z^{-u} T^{v} g, g\right)\right|^{2} d u d v
$$

By (2.2), $c_{g}$ can also be written as

$$
c_{g}=\frac{1}{\|g\|^{2}} \iint\left|\left(Z^{u} E^{-v} \tilde{g}, \tilde{g}\right)\right|^{2} d u d v
$$

which gives

$$
c_{g}=\frac{2 \pi}{\|g\|^{2}} \int_{0}^{\infty} d \omega \int d u e^{u}\left|\tilde{g}(\omega) \tilde{g}\left(e^{u} \omega\right)\right|^{2}=2 \pi \int_{0}^{\infty} \frac{|\tilde{g}(\omega)|^{2}}{\omega} d \omega
$$

3.3. The -transform. Let $g$ be a fixed admissible analyzing wavelet. For arbitrary real $u, v$, define

$$
g^{(u, v)}=Z^{-u} T^{v} g .
$$

For every $h \in \mathrm{H}^{2}$, define the function $e h$ of variables $u, v$ by

$$
\left(e_{h}\right)(u, v)=\frac{1}{\sqrt{c_{g}}}\left(g^{(u, v)}, h\right)
$$

i.e.

$$
(\bigodot h)(u, v)=\frac{1}{\sqrt{c_{g}}} e^{u / 2} \int \bar{g}\left(e^{u} t-v\right) h(t) d t .
$$

In words: $(\mathrm{C} h)(u, v)$ is obtained by "testing" the function $h$ with the help of dilated and shifted analyzing wavelet. The dilation parameter is $u$, and the shift parameter is $v$. The result of testing is multiplied by a normalization factor which depends on the choice of the admissible analyzing wavelet.

We call $C h$ the $e$-transform of $h$ (with respect to $g$ ). By (2.4) we have, with $s(t)=\operatorname{Re} h(t)$,

$$
\left(e_{h}\right)(u, v)=\frac{2}{\sqrt{c_{g}}} e^{u / 2} \int \bar{g}\left(e^{u} t-v\right) s(t) d t
$$


Alternative ways of writing $(e h)(u, v)$ are, by $(2.2),(2.3)$,

$$
\begin{aligned}
(e h)(u, v) & =\frac{1}{\sqrt{c_{g}}}\left(Z^{-u} T^{v} g, h\right)=\frac{1}{\sqrt{c_{g}}}\left(Z^{u} E^{-v} \tilde{g}, \tilde{h}\right) \\
& =\frac{1}{\sqrt{c_{g}}} e^{-u / 2} \int_{0}^{\infty} \overline{\tilde{g}}\left(e^{-u} \omega\right) \tilde{h}(\omega) \exp \left[i v e^{-u} \omega\right] d \omega
\end{aligned}
$$

From (3.7) one sees that

$$
|(e h)(u, v)| \leq \frac{1}{\sqrt{c_{g}}}\|h\|\|g\|
$$

for all $u, v$.

The correspondence $h \rightarrow C h$ is linear.

3.4. Isometry of $\mathcal{C}$. We claim: For every $h \in \mathbf{H}^{2}$, the function $(\mathcal{C} h)(u, v)$ is square integrable, and

$$
\iint|(e h)(u, v)|^{2} d u d v=\|h\|^{2}
$$

Proof. (i) The equality (3.8) holds for $h=g$, since, by (3.5),

$$
\iint|(\circlearrowright g)(u, v)|^{2} d u d v=\frac{1}{c_{g}} \iint\left|\left(Z^{-u} T^{v} g, g\right)\right|^{2} d u d v=\frac{c_{g}}{c_{g}}\|g\|^{2} \text {. }
$$

(ii) Equation (3.8) also holds for every $h$ of the form $h=Z^{-u_{0}} T^{v_{0}} \mathrm{~g}$. Indeed,

$$
\begin{aligned}
(\bigodot h)(u, v) & =\frac{1}{\sqrt{c_{g}}}\left(Z^{-u} T^{v} g, Z^{-u_{0}} T^{v_{0}} g\right)=\frac{1}{\sqrt{c_{g}}}\left(T^{v_{0}} Z^{u_{0}-u} T^{v} g, g\right) \\
& =\frac{1}{\sqrt{c_{g}}}\left(Z^{u_{0}-u} T^{v-v_{0} \exp \left(u-u_{0}\right)} g, g\right) \\
& =\left(\bigodot_{g}\right)\left(u-u_{0}, v-v_{0} e^{u-u_{0}}\right),
\end{aligned}
$$

by (2.1), (3.5) and (3.7).

Now

$$
\begin{aligned}
\iint\left|\left(e_{g}\right)\left(u-u_{0}, v-v_{0} e^{u-u_{0}}\right)\right|^{2} d u d v & =\iint\left|\left(e_{g}\right)\left(u^{\prime}, v-v_{0} e^{u^{\prime}}\right)\right|^{2} d u^{\prime} d v \\
& =\iint\left|\left(e_{g}\right)\left(u^{\prime}, v^{\prime}\right)\right|^{2} d u^{\prime} d v^{\prime}=\|g\|^{2}
\end{aligned}
$$

with $u^{\prime}=u-u_{0}, v^{\prime}=v-v_{0} e^{u-u_{0}}$.

(iii) By standard arguments, (3.8) is extended to all finite linear combinations of vectors of the form $Z^{u} T^{v}$. By irreducibility $\left(\$ 2.3\right.$.), these vectors are dense in $\mathbf{H}^{2}$, and so (3.8) is extended by continuity to all of $\mathbf{H}^{2}$. This completes the proof.

By the polarization identity, one has

$$
\left(C h_{1}, e h_{2}\right)_{L_{2}\left(\mathbb{R}^{2}, d u d v\right)}=\left(h_{1}, h_{2}\right)
$$

for all $h_{1} \in \mathbf{H}^{2}, h_{2} \in \mathbf{H}^{2}$. 
3.5. Inversion of $e$. We now sketch a verification of the fact that $e$, considered as an integral transform, is self-reciprocal. In other words: If $C h$ is given by (3.6), then $h$ can be recovered from $e h$ through the formula

$$
h(t)=\frac{1}{\sqrt{c_{g}}} \iint e^{u / 2} g\left(e^{u} t-v\right)\left(e_{h}\right)(u, v) d u d v .
$$

Since the integral (3.10) cannot converge for every $h$ and every $t$, the formula (3.10) has a sense that is familiar from the $L^{2}$-theory of Fourier transforms or from [1].

In order to obtain (3.10) we write, with a slight stretching of notations, and using (2.4), (3.9),

$$
h\left(t_{0}\right)=\left(\delta_{t_{0}}, h\right)_{L_{2}(\mathbb{R}, d t)}=\frac{1}{2}\left(\delta_{t_{0}}^{(+)}, h\right)_{\mathbf{H}^{2}}=\frac{1}{2}\left(e \delta_{t_{0}}^{(+)}, C h\right)_{L_{2}\left(\mathbb{R}^{2}, d u d v\right)}
$$

where $\delta_{t_{0}}(t)=\delta\left(t-t_{0}\right)$ (Dirac measure) and $\delta^{(+)}(t)=\delta\left(t-t_{0}\right)+(i / \pi) P /\left(t-t_{0}\right)$ (principal part). The function $\left(e \delta_{t_{0}}^{(+)}\right)(u, v)$ can be found by using $(2.4)$ :

$$
\begin{aligned}
\left(e \delta_{t_{0}}^{(+)}\right)(u, v) & =\frac{1}{\sqrt{c_{g}}} e^{u / 2} \int \bar{g}\left(e^{u} t-v\right) \delta_{t_{0}}^{(+)}(t) d t \\
& =2 \frac{1}{\sqrt{c_{g}}} e^{u / 2} \int \bar{g}\left(e^{u} t-v\right) \delta\left(t-t_{0}\right) d t=2 \frac{1}{\sqrt{c_{g}}} e^{u / 2} \bar{g}\left(e^{u} t_{0}-v\right) .
\end{aligned}
$$

Inserting (3.12) into (3.11) gives (3.10).

Remark on redundancy. Equation (3.10) is a way of recovering the function $h(t)$ (and $s(t)=\operatorname{Re} h(t))$ from the function $(\mathrm{e} h)(u, v)$. The function $h(t)$ can also be recovered from values of $(C h)(u, v)$ on suitable subsets of the plane, e.g. from the function

$$
\left(C_{h}\right)(0, v)=\frac{1}{\sqrt{c_{g}}} \int_{0}^{\infty} \overline{\tilde{g}}(\omega) \tilde{h}(\omega) e^{i v \omega} d \omega .
$$

We see that $\tilde{h}(\omega)$ can be obtained from $(\varrho h)(0, v)$ through Fourier transformation and division by $\bar{g}(\omega)$. The last step, however, corresponds-at best-to an unbounded operator. This makes the recovery of $h(t)$ from $(\varrho h)(0, v)$ an impractical proposition in general, and shows the advantage of working with the isometric transformation (3.10) or with suitable discrete approximations to it.

Covariance of $\mathcal{C}$. By the construction of $\mathcal{C}$, we have: If $h_{1}=Z^{u_{1}} h$, then

$$
\left(e_{h_{1}}\right)(u, v)=\left(e_{h}\right)\left(u+u_{1}, v\right) \text {. }
$$

If $h_{2}=T^{v_{2}} h$, then

$$
\left(e h_{2}\right)(u, v)=(e h)\left(u, v-v_{2} e^{u}\right) .
$$

3.6. Reproducing equation. The range of $e$ is not all of $L_{2}\left(\mathbb{R}^{2}, d u d v\right)$. In this section we derive a condition that has to be satisfied by all functions of the form $C h$, with $h \in \mathbf{H}^{2}$. More specific results are given in $\S 4$, for a particular analyzing wavelet.

Define a kernel $G\left(u, v ; u^{\prime}, v^{\prime}\right)$ by

$$
\begin{aligned}
G\left(u, v ; u^{\prime}, v^{\prime}\right) & =\left(g^{(u, v)}, g^{\left(u^{\prime}, v^{\prime}\right)}\right)=\left(Z^{-u} T^{v} g, Z^{-u^{\prime}} T^{v^{\prime}} g\right) \\
& =\left(Z^{u} E^{-v} \tilde{g}, Z^{u^{\prime}} E^{-v^{\prime}} \tilde{g}\right)=\left(Z^{u_{1}} E^{-v_{1}} \tilde{g}, \tilde{g}\right)
\end{aligned}
$$


with

$$
u_{1}=u-u^{\prime} \text { and } \quad v_{1}=v-v^{\prime} e^{u-u^{\prime}}
$$

Then a function $f(u, v)$ that belongs to the range of $C$ must satisfy

$$
f(u, v)=\frac{1}{c_{g}} \iint G\left(u, v ; u^{\prime}, v^{\prime}\right) f\left(u^{\prime}, v^{\prime}\right) d u^{\prime} d v^{\prime}
$$

Indeed, by the definition and isometry of $e$,

$$
\begin{aligned}
(\bigodot h)(u, v) & =\frac{1}{\sqrt{c_{g}}}\left(g^{(u, v)}, h\right)=\frac{1}{\sqrt{c_{g}}}\left(\bigodot^{(u, v)}, e_{h}\right) \\
& =\frac{1}{\sqrt{c_{g}}} \iint\left(\overline{\bigodot^{(u, v)}}\right)\left(u^{\prime}, v^{\prime}\right)\left(\bigodot_{h}\right)\left(u^{\prime}, v^{\prime}\right) d u^{\prime} d v^{\prime} .
\end{aligned}
$$

Now

$$
\left(\bigodot g^{(u, v)}\right)\left(u^{\prime}, v^{\prime}\right)=\frac{1}{\sqrt{c_{g}}}\left(g^{\left(u^{\prime}, v^{\prime}\right)}, g^{(u, v)}\right)
$$

giving (3.15).

\subsection{Cycle-octave representations.}

THEOREM. Let $s(t)$ be any real-valued square integrable function, and $g$ an admissible analyzing wavelet. Associate to $s$ the function $S(u, \tau)$ defined by

$$
S(u, \tau)=\frac{2}{\sqrt{c_{g}}} \int \bar{g}\left(e^{u} t-e^{-u} \tau\right) s(t) d t .
$$

Then $s(t)$ can be recovered from $S(u, \tau)$ through

$$
s(t)=\operatorname{Re} h(t)
$$

where

$$
h(t)=\frac{1}{\sqrt{c_{g}}} \iint g\left(e^{u} t-e^{-u} \tau\right) S(u, \tau) d u d \tau
$$

One has

$$
\iint|S(u, \tau)|^{2} d u d \tau=2 \int s(t)^{2} d t
$$

The function $h(t)$ defined by (3.18) belongs to $\mathbf{H}^{2}$.

An approximate discrete version of (3.16), (3.18), was discovered by one of us [7].

The statements of this theorem are an immediate consequence of the results proved so far, if we introduce the variable

$$
\tau=e^{u} v
$$


3.8. Group-theoretical comments. The objects that we study, namely

$$
\sqrt{c_{g}}(e h)(u,-v)=\left(g, T^{v} Z^{u} h\right)
$$

are matrix elements (coefficients, in another terminology) of the irreducible representation, in $\mathbf{H}^{2}$, of the two-parameter group of shifts and dilations. We have shown that these coefficients, considered as functions on the group, are square integrable with respect to the right Haar measure $d x_{R}=d u d v$, if the vector $g$ is suitably chosen.

If the standard theory of square integrable representations were applicable here (see e.g. [2]), it would follow that all coefficients of this representation are square integrable i.e. that all wavelets are admissible. However, the standard theory holds only for unimodular groups (i.e. groups possessing a right- and left-invariant Haar measure), while the group here is the prime example of nonunimodularity. (The left-invariant Haar measure is $d x_{L}=e^{-u} d u d v$ ). Our results fit into the general theory of square integrable representations of nonunimodular groups, developed by Duflo and Moore [3].

\section{The -transform: wavelet $g_{\alpha}$.}

4.1. The function $\tilde{\boldsymbol{g}}_{\alpha}$. Among all admissible wavelets there is one that plays-in the $\mathbf{H}^{2}$-theory that we are concerned with-the same privileged role that the Gaussian plays in $L^{2}$-theory. The Fourier transform of this wavelet is just the image of a Gaussian under a natural map.

Let $\alpha>0$. Consider the function $\tilde{g}_{\alpha}(\omega)$ defined by

$$
\tilde{g}_{\alpha}(\omega)= \begin{cases}\exp \left(-\frac{\alpha}{2} \ln ^{2} \omega\right) & \text { for } \omega>0 \\ 0 & \text { for } \omega \leq 0\end{cases}
$$

Notice that $\tilde{g}_{\alpha}(\omega)$ is infinitely differentiable everywhere, in particular at $\omega=0$. Furthermore, $\tilde{g}_{\alpha}(\omega)$ tends to zero at infinity faster than any inverse polynomial.

We shall first verify that $g_{\alpha}$ is admissible, by using the criterion (3.2): one has

$$
\int_{0}^{\infty}\left|\tilde{g}_{\alpha}(\omega) \tilde{g}_{\alpha}\left(e^{u} \omega\right)\right|^{2} d \omega=\sqrt{\frac{\pi}{2 \alpha}} e^{1 / 8 \alpha} e^{-\alpha u^{2} / 2-u / 2}
$$

and consequently

$$
\int d u e^{u} \int_{0}^{\infty}\left|\tilde{g}_{\alpha}(\omega) \tilde{g}_{\alpha}\left(e^{u} \omega\right)\right|^{2} d \omega=\frac{\pi}{\alpha} e^{1 /(4 \alpha)}
$$

which shows admissibility, and also gives

$$
c_{g}=2 \pi^{3 / 2} \alpha^{-1 / 2} \text {. }
$$

The basic functional equation satisfied by $\tilde{g}_{\alpha}(\omega)$ is

$$
\left(Z^{u} \tilde{g}_{\alpha}\right)(\omega)=e^{-\alpha u^{2} / 2-u / 2} \omega^{\alpha} \tilde{g}_{\alpha}(\omega)
$$

It corresponds to the equation relating a shifted Gaussian to the Gaussian multiplied by an exponential. 


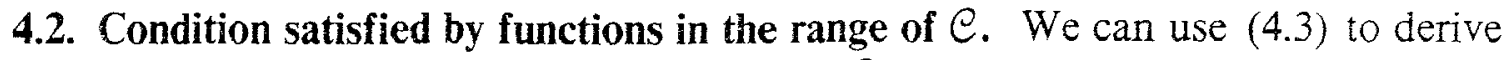

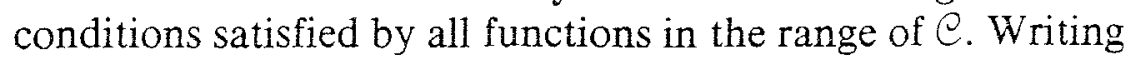

$$
(\bigodot h)(u, v)=\frac{1}{\sqrt{c_{g}}}\left(Z^{u} E^{-v} \tilde{g}_{\alpha}, \tilde{h}\right)=\left(E^{-v \exp (-u)} Z^{u} \tilde{g}_{\alpha}, \tilde{h}\right) \frac{1}{\sqrt{c_{g}}}
$$

and evaluating $Z^{u} \tilde{g}$ by (4.3), we obtain

$$
(\circlearrowright h)(u, v)=\frac{1}{\sqrt{c_{g}}} e^{-\alpha u^{2} / 2-u / 2} \int_{0}^{\infty} \exp \left(i v e^{-u} \omega\right) \omega^{\alpha u} \overline{\tilde{g}}(\omega) \tilde{h}(\omega) d \omega .
$$

Introducing variables

$$
z=\alpha u-1 \text { and } q=v e^{-u}
$$

and writing

$$
\Psi(z, q)=\left(e_{h}\right)(u, v)
$$

we see that

$$
\Psi(z, q)=\frac{1}{\sqrt{c_{g}}} e^{-\left(z^{2}-z\right) / 2 \alpha} \int_{0}^{\infty} e^{i q \omega} \omega^{z-1} \overline{\tilde{g}}_{\alpha}(\omega) \tilde{h}(\omega) d \omega
$$

It follows from (4.4) that $\Psi(z, q)$ satisfies

$$
\frac{\partial \Psi}{\partial q}=i e^{z / \alpha} \Psi(z+1, q)
$$

and, more generally

$$
\Psi(z+n, q)=(-i)^{n} e^{-(n z+(n-1) n) / 2 \alpha} \frac{\partial^{n} \Psi}{\partial q^{n}} .
$$
form

4.3. The function $\Gamma_{\alpha}(z)$. The wavelet $g_{\alpha}(t)$ is given by the inverse Fourier trans-

$$
g_{\alpha}(t)=(2 \pi)^{-1 / 2} \int_{0}^{\infty} e^{i \omega t} \tilde{g}_{\alpha}(\omega) d \omega
$$

which does not seem expressible in closed form through special functions known to us. In order to evaluate it and related quantities we have found it convenient to introduce the function $\Gamma_{\alpha}(z)$ which will now be discussed and which, we believe, is also intrinsically interesting.

If $\alpha>0$ and if $z=x+i y$ is arbitrary complex, define $\Gamma_{\alpha}(z)$ by

$$
\Gamma_{\alpha}(z)=\int_{0}^{\infty} \omega^{z-1} e^{-\omega} \exp \left(-\frac{\alpha}{2} \ln ^{2} \omega\right) d \omega
$$

This definition is modeled on the definition

$$
\Gamma(z)=\int_{0}^{\infty} \omega^{z-1} e^{-\omega} d \omega \quad(\operatorname{Re} z>0)
$$

of Euler's gamma function. Because of the factor $\exp \left(-\alpha \ln ^{2} \omega / 2\right)$, the function $\Gamma_{\alpha}(z)$ is entire analytic in $z$, in contrast to $\Gamma(z)$. If the factor $\exp \left(-\alpha \ln ^{2} \omega / 2\right)$ were replaced by a step function, the resulting integral would be an incomplete gamma function. 
The substitution $\omega=e^{s}$ brings $\Gamma_{\alpha}(z)$ to the form

$$
\Gamma_{\alpha}(z)=\int_{-\infty}^{\infty} e^{z s} \exp \left(-e^{s}-\frac{\alpha}{2} s^{2}\right) d s
$$

We may think of $\Gamma_{\alpha}(z)$ as being a hybrid between a Gaussian and the $\Gamma$-function. This is made precise e.g. through the following statement:

If $\operatorname{Re} z>0$, then

$$
\Gamma_{\alpha}(z)=\frac{1}{\sqrt{2 \pi \alpha}} \int_{-\infty}^{\infty} e^{-u^{2} / 2 \alpha} \Gamma(z-i u) d u .
$$

The function $\Gamma_{\alpha}(z)$ satisfies a functional equation that goes over into the classical $\Gamma(z)=(z-1) \Gamma(z-1)$ in the limit $\alpha \rightarrow 0$. Denote by $\Gamma_{\alpha}^{(n)}$ the $n$th derivative of $\Gamma_{\alpha}$ with respect to $z$. We have then

$$
\Gamma_{\alpha}(z)=(z-1) \Gamma_{\alpha}(z-1)-\alpha \Gamma_{\alpha}^{(1)}(z-1)
$$

and, more generally,

$$
\Gamma_{\alpha}^{(n)}(z)=(z-1) \Gamma_{\alpha}^{(n)}(z-1)-\alpha \Gamma_{\alpha}^{(n+1)}(z-1)+n \Gamma_{\alpha}^{(n-1)}(z-1) \quad(n=1,2, \cdots) .
$$

The function $\Gamma_{\alpha}(z)$ has asymptotic expansions for large $|z|$. For instance, we shall write the analogue of the formula $\Gamma(z) \simeq(2 \pi)^{1 / 2} e^{-z+(z-1 / 2) \ln z}(1+1 / 12 z)$ :

Denote by $z_{1}$ the solution of

$$
z_{1}=z-\alpha \ln z_{1}
$$

which is positive when $z$ is large and positive. Then

$$
\Gamma_{\alpha}(z) \simeq \sqrt{\frac{2 \pi}{z_{1}+\alpha}} e^{-z_{1}+\left(z^{2}-z_{1}^{2}\right) / 2 \alpha}\left[1-\frac{z_{1}}{8\left(z_{1}+\alpha\right)^{2}}+\frac{5 z_{1}}{24\left(z_{1}+\alpha\right)^{3}}\right] .
$$

The expression (4.10) is numerically quite accurate even for small values of $|z|$.

If for fixed $y, x$ is let go to $-\infty$, one has

$$
\Gamma_{\alpha}(x+i y) \sim \sqrt{\frac{2 \pi}{\alpha}} e^{(x+i y)^{2} / 2 \alpha} .
$$
satisfies

We consider next the variation of $\Gamma_{\alpha}(z)$ with $\alpha$. From (4.6) we see that $\Gamma_{\alpha}(z)$

$$
\frac{\partial \Gamma_{\alpha}}{\partial \alpha}=-\frac{1}{2} \frac{\partial^{2} \Gamma_{\alpha}}{\partial z^{2}}
$$

Notice the minus sign in (4.11). We obtain the usual heat equation if we consider $\Gamma_{\alpha}(x+i y)$ as function of $y$ for fixed $x$ :

$$
\frac{\partial \Gamma_{\alpha}(x+i y)}{\partial \alpha}=\frac{1}{2} \frac{\partial^{2} \Gamma_{\alpha}(x+i y)}{\partial y^{2}} .
$$

The function $\Gamma_{\alpha}(x+i y)$ is bounded by its values on the real axis: $\left|\Gamma_{\alpha}(x+i y)\right|<\Gamma_{\alpha}(x)$. Along parallels to the imaginary axis, it decreases faster than any inverse polynomial. Considered as a function of $\alpha, \Gamma_{\alpha}(x)$ is monotonically decreasing. On the positive real 
axis, $\Gamma_{\alpha}(x)$ is bounded by Euler's $\Gamma$-function to which it tends as $\alpha \rightarrow 0$. For every $x, y$, we have $\left|\Gamma_{\alpha}(x+i y)\right|<\sqrt{2 \pi / \alpha} e^{x^{2} / 2 \alpha}$. If we let $\alpha$ tend to $+\infty$ while keeping $z$ fixed, then $\Gamma_{\alpha}(z)$ behaves as $\sqrt{2 \pi / \alpha} e^{\alpha z^{2} / 2}$.

Details and further results will be given elsewhere.

4.4. A class of integrals. With the help of the function $\Gamma_{\alpha}(z)$ one can evaluate integrals of the form

$$
h_{\alpha, \beta}(q)=\int_{0}^{\infty} \omega^{\beta-1} \exp \left(-\frac{\alpha}{2} \ln ^{2} \omega\right) e^{i \omega q} d \omega
$$

which will be needed below. Here $\beta$ is complex and arbitrary, $\alpha>0, q \neq 0$, and $\operatorname{Im} q \geq 0$.

It is convenient to introduce the variable

$$
\kappa=\ln \left(\frac{1}{i} q\right)=\ln |q|+i \arg q-i \frac{\pi}{2} .
$$

If $q$ is real, then $\operatorname{Im} \kappa=-(\pi / 2) \operatorname{sgn} q$.

The integral (4.13) is first transformed into

$$
h_{\alpha, \beta}(q)=\int_{-\infty}^{\infty} \exp \left(-e^{\kappa+s}-\frac{\alpha}{2} s^{2}+\beta s\right) d s
$$

by the substitution $\omega=e^{s}$. Then the substitution $s^{\prime}=s+\kappa$ and a shift of the path of integration back to the real axis bring it to the form

$$
h_{\alpha, \beta}(q)=\exp \left(-\frac{\alpha}{2} \kappa^{2}-\beta \kappa\right) \int \exp \left[-e^{s}+(\alpha \kappa+\beta) s-\frac{\alpha}{2} s^{2}\right] d s
$$

giving the result

$$
h_{\alpha, \beta}(q)=\exp \left(-\frac{\alpha}{2} \kappa^{2}-\beta \kappa\right) \Gamma_{\alpha}(\alpha \kappa+\beta)
$$

Remarks. The value of the integral (4.13) for $q=0$ can be computed directly. It is

$$
h_{\alpha, \beta}(0)=\sqrt{\frac{2 \pi}{\alpha}} \exp \left(\frac{\beta^{2}}{2 \alpha}\right) \text {. }
$$

The function $h_{\alpha, \beta}(q)$ defined by (4.15) and (4.16) is infinitely differentiable on the real axis, and it decreases at infinity faster than any inverse polynomial. Furthermore, $h_{\alpha, \beta}$ belongs to $\mathbf{H}^{2}$.

As a function of $\beta, h_{\alpha, \beta}$ is entire analytic, and square integrable on every parallel to the imaginary axis. We have, from (4.13)

$$
\frac{d^{n}}{d q^{n}} h_{\alpha, \beta}=i^{n} h_{\alpha, \beta+n}
$$

4.5. Explicit expressions. We can now write down expressions for various quantities of interest:

1) The wavelet $g_{\alpha}(t)$ is given by

$$
g_{\alpha}(t)=(2 \pi)^{-1 / 2} e^{-\alpha \theta^{2} / 2-\theta} \Gamma_{\alpha}(\alpha \theta+1) \quad\left(\theta=\ln \left(\frac{1}{i} t\right)\right) .
$$




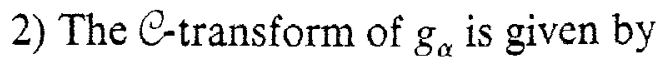

$$
\left(\bigodot g_{\alpha}\right)(u, v)=2^{-1 / 2} \pi^{-3 / 4} \alpha^{1 / 4} e^{-u^{2} \alpha / 4} e^{-\alpha \eta^{2}-\eta} \Gamma_{2 \alpha}(2 \alpha \eta+1)
$$

where $\eta=\ln \left((1 / i) v e^{-u / 2}\right)$.

3) The kernel of the integral equation satisfied by the functions in the range of $e$ is (compare (3.15))

$$
G\left(u, v ; u^{\prime}, v^{\prime}\right)=e^{-\alpha u_{1}^{2} / 4-\alpha \eta_{1}^{2}-\eta_{1}} \Gamma_{2 \alpha}\left(2 \alpha \eta_{1}+1\right)
$$

where $u_{1}=u-u^{\prime}$ and

$$
\eta_{1}=\ln \left(\frac{1}{i} v e^{\left(u-u^{\prime}\right) / 2}-\frac{1}{i} v^{\prime} e^{-\left(u-u^{\prime}\right) / 2}\right) .
$$

Acknowledgments. One of us (A. G.) would like to thank H. Bacry for stimulating conversations.

\section{REFERENCES}

[1] V. Bargmann, On a Hilbert space of analytic functions and an associated integral transform, Part I, Comm. Pure Appl. Math, 14 (1961), pp. 187-214; Part II, ibid. 20 (1967), pp. 1-101.

[2] J. Dixmier, Les $C^{*}$-algèbres et leurs représentations, Gauthier-Villars, Paris, 1969.

[3] M. Duflo AND C. C. MOORE, On the regular representation of a nonunimodular locally compact group, J. Funct. Anal., 21 (1976), pp. 209-243.

[4] D. GABOR, Theory of Communication, J. Inst. Electr. Engin. (London) 93 (III), 1946, pp. 429-457.

[5] C. W. HeLstrom, An expansion of a signal in Gaussian elementary signals, IEEE Trans. Infor. Theory, IT 12 (1966), pp. 81-82.

[6] J. R. Klauder AND E. C. Sudarshan, Fundamentals of Quantum Optics, Benjamin, New York, 1968.

[7] J. Morlet, G. Arens, E. Fourgeau AND D. Giard, Wave propagation and sampling theory, Part II, Geophys. 47 (1982), pp. 222-236.

[8] J. MORLET, Sampling theory and wave propagation, Proc. 51st Annual International Meeting of the Society of Exploration Geophysicists, Los Angeles, 1981.

[9] N. YA. VILENKIN, Special Functions and the Theory of Group Representations, American Mathematical Society, Providence, RI, 1968. 